\title{
Dynamic Network Selection in Vehicular Heterogeneous Wireless Networks
}

\author{
Lang Gaiping ${ }^{1, a}$, Xu Yubin ${ }^{1, b}$ and Ma Lin ${ }^{1, c}$
}

\author{
${ }^{1}$ School of Electronic and Information Engineering, Harbin Institute of Technology, Harbin 150008, \\ China \\ alanggaiping@163.com, bybxu@hit.edu.cn, ${ }^{c}$ malin@hit.edu.cn
}

Keywords: Intelligent Transportation Systems (ITS); LTE; WLAN; Radio Resource

\begin{abstract}
Three general types of applications, safety applications, traffic applications and non-safety applications, are developed over vehicular networks. Different usage frequency may occur for different applications in vehicular networks. Most of current researches only focus on the safety applications, traffic applications or non-safety applications. Actually, these applications are used by one vehicular according to his dynamic requirements. So, this paper takes these applications together into account, and corresponding access policy is proposed.
\end{abstract}

\section{Introduction}

In the past, cars embedded with advanced materials and sensors to strengthen safety. In recent years, with the development of wireless communication technologies, cars are equipped with radio interfaces. Using these radio interfaces, they can not only obtain safe guarantee but also enjoy other new applications. Three general types of applications are developed over vehicular networks. Safety applications improve the safety of the passengers on the roads through sending warning information to their neighboring vehicles about accident, slippery road and emergency vehicle warning and so on. Traffic applications provide drivers with the traffic situation and road information to avoid congestion. Non-safety applications include many types of services, such as video conference, web browning, map and weather information downloading and so on.

Nowadays, various wireless access technologies, such as Wireless Local Access Networks (WLAN) and cellular networks (3G, LTE), are coexisting. In this heterogeneous environment, different networks have different characteristics, and can meet diverse quality of service (QoS) demands of end users. None of these networks, however, satisfies all the required services and applications. If these networks with compensatory characteristics can cooperate, users with multiple radio interfaces may experience satisfied services at anytime and anywhere. Meanwhile, cooperative use of the multiple radios enhances the performance of the heterogeneous system in the sense that the network resources can be used efficiently. So, in this paper we use heterogeneous framework to develop Intelligent Transportation Systems (ITS).

\section{Related Works}

Recently, researches about vehicular heterogeneous networks are emerging to enhance the vehicle communication quality. Generally, member networks include WLAN, cellular network, and WiMAX and so on. Heterogeneous vehicular network architecture was introduced, and a mobility pattern aware routing protocol was proposed to provide the reliable route path for $\mathrm{V} 2 \mathrm{~V}$ communication [1]. Emergency services and road safety evolves with the development of vehicular communication networks [2]. Most of the researches about vehicular communications and vehicular networks only deal with a single type of application, such as only emergency services or only safety application. Drivers may need to use various applications, and single type of networks cannot meet all applications' requirements at the same time [3]. So heterogeneous vehicular network is needed, another reason is that WLAN's coverage area is relatively small and not available in every place. In heterogeneous vehicular networks, group handover may occur frequently, passengers travelling by bus or train experience similar channel condition and they are close to each other. If they make 
decision individually, they may select the same network to handover. This may result in congestion and user even may be dropped. Considering characters of vehicular networks, such as high speed, mobility pattern, and topology restrictions, group users are enabled to select network from the point view of the whole system based on mobility prediction [4]. Mobility prediction is an important problem in handover decision, and it can provide useful information for user position and users distribution.

\section{Problem Formulation}

Motivation. A key motivation of considering heterogeneous vehicular network is that WLAN will only be effective when it is ubiquitously deployed and vehicles' speed are relatively low, but this will not happen in these years. Therefore, nowadays a heterogeneous platform is the best way forward. In fact, LTE is a type of wide area network, and WLAN is deployed only in the hotspot. They are complementary in the data rate and coverage area and so on. So for LTE and WLAN, cooperating to provide all kinds of applications for vehicles is a reasonable choice.

Network Architecture. Here, a simple scenario is presented in fig. 1, and heterogeneous networks are composed of WLAN and LTE. We assumed that only one direction of vehicles is considered.

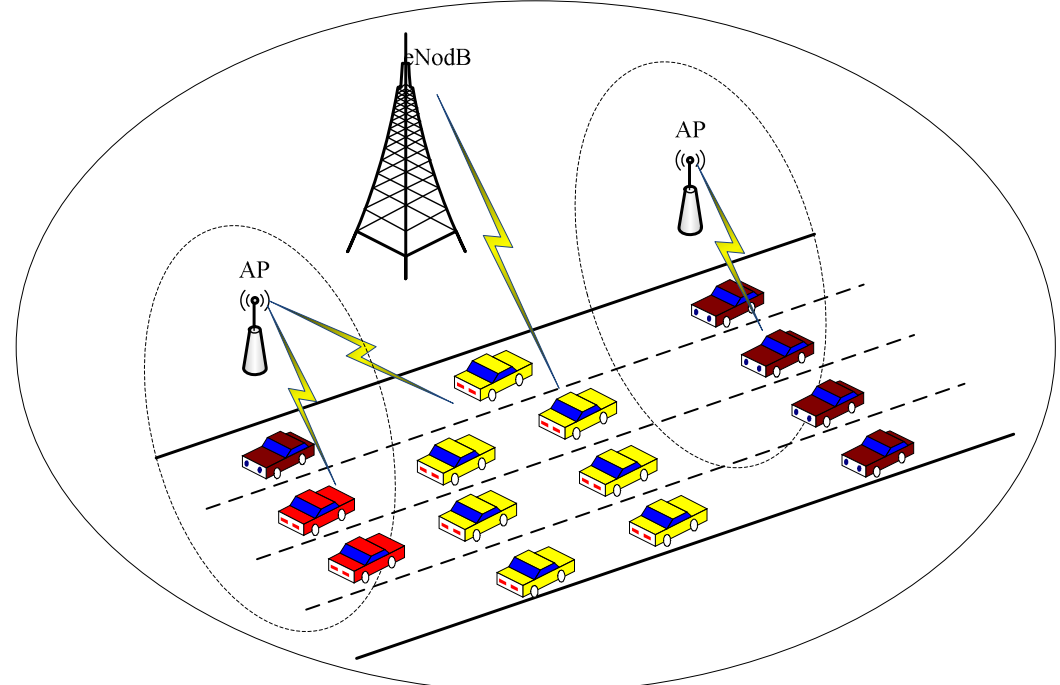

Fig. 1 Network architecture

Access Policy. We consider a highway vehicular communication scenario, where without loss of generality we can confine our analysis in spatial domain to the direction of moving vehicles. 802.11e (WLAN) has two access mechanisms [5], EDCA and HCCA. HCCA has higher priority than EDCA. So, if one vehicle is using WLAN now, he will choose appropriate access mechanism according to the application on use. If he needs to send or receive the safety message, he will use HCCA to compete for channel using. V2R communication is only suit for low speed condition or static environment. V2V is an optional communication. The ability of LTE supporting safety application is poor, but the ability supporting infotainment applications are stronger than WLAN. Because of small coverage area of WLAN, high speed vehicle will encounter frequent handover, which induces more overhead and waste valuable resource. Therefore, on the condition of safety guarantee, satisfying vehicles maximally is a promising issue.

\section{Overview of LTE and WLAN Technology}

LTE. LTE is a standard for wireless data communications technology and an evolution of the GSM/UMTS standards. E-UTRAN is radio network of LTE. It consists of eNBs, providing the E-UTRA user plane and control plane protocol terminations towards the UE. The eNBs are interconnected with each other by means of the X2 interface. The eNBs are also connected by means of the S1 interface to the EPC (Evolved Packet Core), more specifically to the MME (Mobility 
Management Entity) by means of the S1-MME interface and to the Serving Gateway (S-GW) by means of the S1-U interface. The S1 interface supports a many-to-many relation between MMEs / Serving Gateways and eNBs [6]. LTE architecture is in fig. 2.

WLAN. IEEE 802.11 specifies two medium access control mechanisms: the mandatory distributed coordination function (DCF) and the optional point coordination function (PCF) [7]. To enhance QoS support in 802.11, IEEE 802.11e is introduced, and his MAC mechanism is the hybrid coordination function (HCF). The HCF includes two medium access mechanisms: contention-based channel access and controlled channel access, providing prioritized and parameterized QoS access to the wireless medium. The contention-based access method of HCF is the enhanced distributed channel access (EDCA). The EDCA provides QoS support using different access categories (ACs) with their independent back-off entities. The controlled channel access is HCF controlled channel access (HCCA). HCCA provides polled access to the wireless medium.

An AC uses three parameters to indicate his contention process. They are AIFSD[AC], $\mathrm{CWmin}[\mathrm{AC}]$, and $\mathrm{CWmax}[\mathrm{AC}]$. $\mathrm{CW}$ parameter takes an initial value of $\mathrm{CWmin}[\mathrm{AC}]$, and his maximum is $\mathrm{CWmax}[\mathrm{AC}]$. Different $\mathrm{AC}$ have different these three parameters. AIFSD[AC] is calculated as

AIFSD $[\mathrm{AC}]=\mathrm{SIFS}+\mathrm{AIFS}[\mathrm{AC}] \cdot$ SlotTime.

AIFS $[A C]$ is an integer greater than zero. SIFS is Short Inter Frame Space. SlotTime is time units in WLAN.

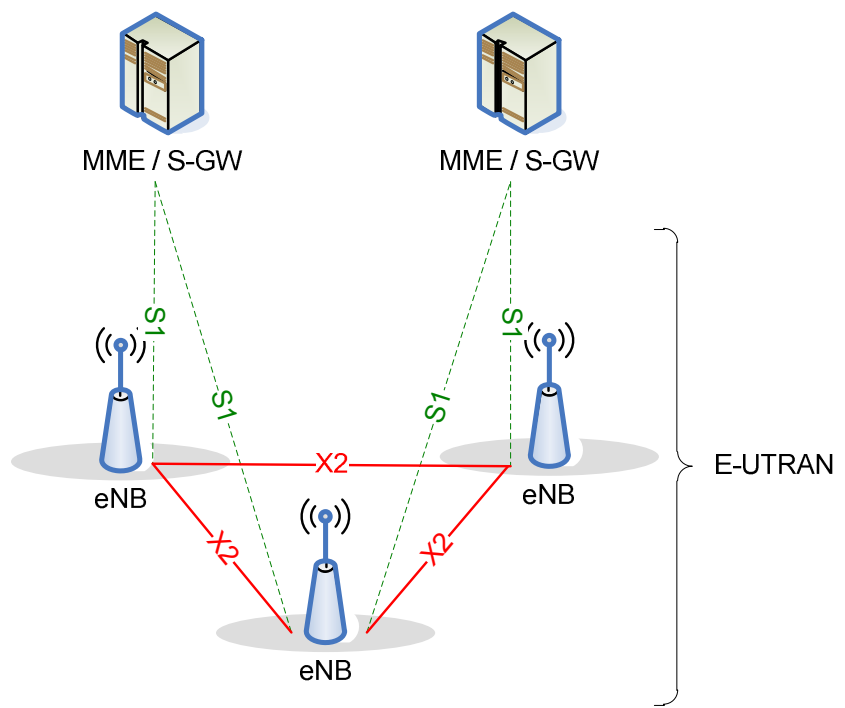

Fig. 2 LTE architecture ${ }^{[6]}$

\section{Communication Process}

In this paper, vehicles select different networks to access according to their speed and application type on use. Through properly selection, drivers can achieve satisfied service. From the discussion of previous section, we present a new network selection way. A vehicle needs to know available networks at any time in order to make decision about whether handover or not. Then according to the current network condition and vehicle's own status, he can start selection process. General process is shown in fig. 3.

For the decision of an available network, vehicle checks received signal strength (RSS), if RSS is higher than RSS threshold, available bandwidth is estimated. If available bandwidth is larger than requirement, this network is considered available. Available bandwidth is calculated

$$
B W=B_{0}-L \frac{N A V}{T_{n}+0.5 T_{n, c}(N-1)}
$$

Where $B_{0}$ is total bandwidth of system, $L$ is mean frame length, $T_{n}$ is NAV duration of a successful transmission, $N$ is retransmission times [8]. 
If the number of available networks is larger than one, the problem can be divided into two sides. If the speed of vehicle is higher than the threshold $V_{t h}$, it need to handover to LTE network, or keep in original network if it is in the LTE network at last moment. If the speed of vehicle is lower than the threshold $V_{t h}$, the vehicle is accessed to WLAN network. But which access mechanism is used is judged according to application type of the vehicle, if it is safety application, HCCA is used, otherwise EDCA is used.

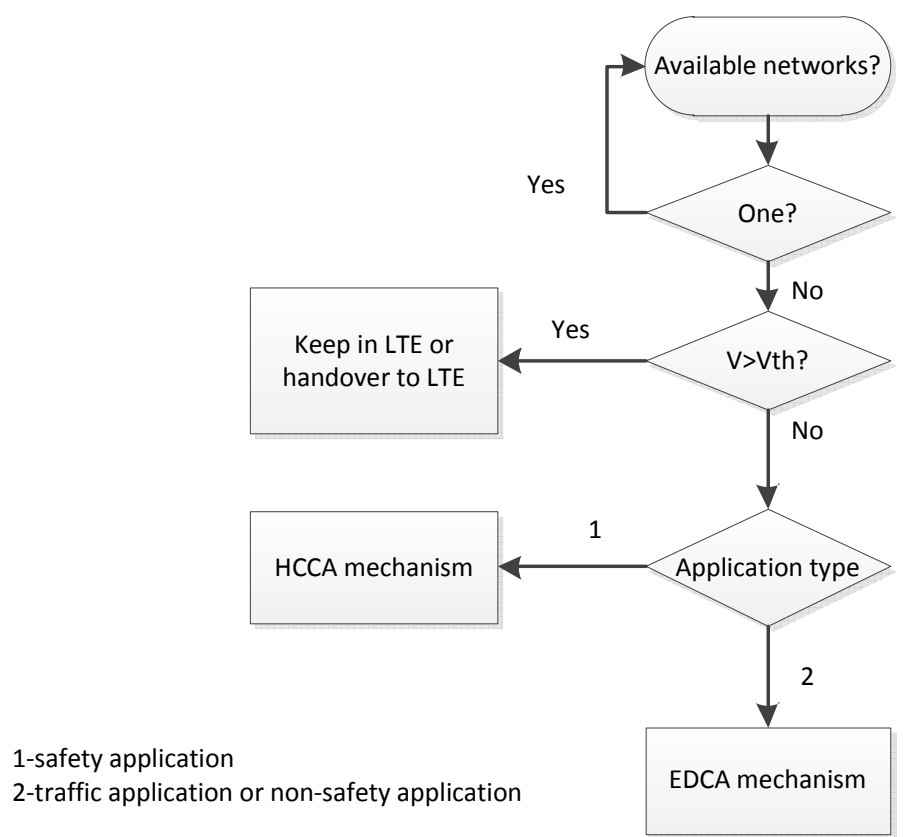

Fig. 3 Flowchart of network selection

\section{Conclusions}

Throughout previous analysis, we can know that vehicles achieved satisfied experience by accessing different networks which are cooperative heterogeneous system composed of WLAN and LTE. Using this network selection algorithm, all applications are considered and correctly processed. Furthermore, system resource is utilized efficiently. Meanwhile, drivers not only achieve safety information in time, but also they can experience good time on the road.

\section{References}

[1] C. C. Hung, H. Chan, and E. H. K. Wu, Mobility pattern aware routing for heterogeneous ve hicular networks, Wireless Communications and Networking Conference (WCNC), Apr. 2008, pp. 2200-2205.

[2] F. J. Martinez, C.K. Toh, J.C. Cano, C. T. Calafate, and P. Manzoni, Emergency Services in Future Intelligent Transportation Systems based on Vehicular Communication Networks, IEEE Intelligent Transportation Systems Magazine, 2010, pp. 6-20.

[3] E. Hossain, G. Chow, V. C. Leung, R. McLeod, J. Misic, V. W. wong, and O. Yang, Vehicular telematics over heterogeneous wireless networks: A survey, Computer Communications, vol. 33, 2010, pp. 775-793.

[4] G.X. Zhang, F.Q. Liu, an Auction Approach to Group Handover with Mobility Prediction in Heterogeneous Vehicular Networks, 11 th International Conference on ITS Telecommunications, 2010, pp. 584-589 
[5] IEEE 802.11e, Part 11: Wireless Medium Access Control (MAC) and Physical Layer (PHY) Speci fi cations: Medium Access Control (MAC) Quality of Service Enhancements, IEEE Std. 802.11e-2005, 2005.

[6] 3GPP TS 36.300 Tech. Spec., Evolved Universal Terrestrial Radio Access (E-UTRA) and Evolved Universal Terrestrial Radio Access Network (E-UTRAN) work in progress, June 2011.

[7] IEEE Std. 802.11, "Wireless LAN Medium Access Control (MAC) and Physi-cal Layer (PHY) Specifications," 2012.

[8] Dong Ma, Student Member, IEEE, and Maode Ma, Senior Member, IEEE, "A QoS Oriented Vertical Handoff Scheme for WiMAX/WLAN Overlay Networks", IEEE Transactions on Parallel and Distributed Systems, VOL. 23, NO. 4, APRIL 2012, pp. 598-606. 